\title{
Is There a Role for Functional MRI for the Assessment of Extracapsular Extension in Prostate Cancer?
}

\author{
METIN SERTDEMIR ${ }^{1 *}$, ANJA M. WEIDNER $^{2 *}$, STEFAN O. SCHOENBERG $^{2}$, \\ JOHN N. MORELLI ${ }^{3}$, AXEL HAECKER ${ }^{4}$, MATTHIAS KIRCHNER ${ }^{5}$, CHRISTEL WEISS ${ }^{6}$, \\ DANIEL HAUSMANN ${ }^{2}$, DIETMAR J. DINTER ${ }^{7}$ and ULRIKE I. ATTENBERGER ${ }^{2}$ \\ ${ }^{1}$ Diagnostische Gemeinschaftspraxis, Karlsruhe, Germany; \\ ${ }^{2}$ Institute of Clinical Radiology and Nuclear Medicine, University Medical Center Mannheim, Mannheim, Germany; \\ ${ }^{3}$ Department of Radiology, St. John's Medical Center, Tulsa, OK, U.S.A.; \\ ${ }^{4}$ Department of Urology, University Medical Center Mannheim, Mannheim, Germany; \\ ${ }^{5}$ Institute of Pathology, University Medical Center Mannheim, Mannheim, Germany; \\ ${ }^{6}$ Department of Biostatistics, University Medical Center Mannheim, Mannheim, Germany; \\ ${ }^{7}$ Radiologie Schwetzingen, Schwetzingen, Germany
}

\begin{abstract}
Background/Aim: Exctracapsular extension (ECE) in prostate cancer has a high impact on treatment decision. MRI might predict presence of ECE non-invasively. Patients and Methods: Triplanar T2w-sequences, DWI (diffusion weighted imaging) and DCE (dynamic contrast-enhanced imaging) of 34 patients with $\mathrm{PCa}$ were analyzed to prior prostatectomy. Sensitivity (SS) and specificity (SP) of T2w, apparent diffusion coefficient $(A D C)$, plasma flow $(P F)$ and mean transit time (MTT) normalized by PCa/normal tissue ratio for prediction of $C I$ (capsular infiltration)/ECE were determined by area-underthe-receiver-operating-characteristics analysis. Results: SS/SP for detecting ECE was 29/85. AUC (area under the curve) of ECE cases was 0.98/0.92/0.69 (cut-off-ratios 3.2/0.51/0.46), SS 93/100/86\% and SP 95/80/50\% for PF-/MTT-/ADC-ratios, respectively. $P F$ - and MTT-ratios between $C I$ and without $C I / E C E$ differed significantly $(P F, p<0.0001 ; M T T, p=0.0134)$ with SS/SP 84/89\% for PF and SS/SP 52/100\% for MTT-ratios. No significant differences regarding $A D C$-ratios were identified. Conclusion: ECE/CI can be assessed by quantitative DCE analysis with great diagnostic confidence and higher specificity than $A D C$.
\end{abstract}

\footnotetext{
*These Authors contributed equally to this study.

Correspondence to: Weidner Anja, Institute of Clinical Radiology and Nuclear Medicine, University Medical Center Mannheim, TheodorKutzer-Ufer 1-3, 68167 Mannheim, Germany. Tel: +49 6213832067, Fax: +49 6213831910, e-mail: anja.weidner@medma.uni-heidelberg.de

Key Words: Magnetic resonance imaging, prostate cancer, dynamic contrast-enhanced MRI, diffusion weighted MRI, extracapsular extension.
}

Prostate cancer (PCa) remains a major health concern among men in both developing and developed countries. In 2013, approximately 3 million men were living with a history of $\mathrm{PCa}$ in the United States, and an additional 233,000 cases were diagnosed in 2014 (1). Nerve-sparing radical prostatectomy (RP) is the treatment standard for clinically localized PCa, especially in low- and intermediate-risk patients $(2,3)$. In highrisk patients, treatment options also include beside surgery RT (radiation therapy), hormonal therapy, or a combination (4). The choice of the treatment strategy depends on cancer stage and grade, as well as patient comorbidity and age. The presence of extracapsular extension (ECE) and seminal-vesicleinfiltration (SVI) also has a significant impact, as patients presenting with ECE or SVI are not eligible for RP (5). Moreover, ECE, which is poorly detected by standard clinical tests, can appear in early stages of PCa with an incidence of up to $60 \%$ in patients with clinical stage $\mathrm{T} 1 / \mathrm{T} 2 \mathrm{PCa}(6)$.

Due to its excellent intrinsic soft-tissue-contrast and ability to provide functional and morphologic information within one examination, Magnetic Resonance Imaging (MRI) has evolved into an established tool for the diagnostic evaluation of PCa. T2w-sequences are most commonly used for the assessment of ECE. However, studies have found significant variation in the accuracy of $\mathrm{T} 2 \mathrm{w}$-sequences for the detection of ECE and SVI with reported values ranging between $50 \%$ and $92 \%$ depending upon reader experience $(7,8)$.

In this context, functional MRI techniques such as dynamic-contrast-enhanced (DCE) and diffusion-weighted (DWI) MRI are increasingly utilized to improve the accuracy of ECE detection. DCE-MRI allows a non-invasive evaluation of tumor perfusion and vascular permeability measuring the changes in gadolinium concentration in blood and tissue over time. The differences in signal-intensity-time 
Table I. Scan parameters of the T2 weighted, DCE MRI and DWI MRI sequences.

\begin{tabular}{|c|c|c|c|}
\hline & \multicolumn{3}{|c|}{$\mathrm{T} 2 \mathrm{~W}$} \\
\hline & axial/sagittal/coronal & DCE & DWI \\
\hline $\mathrm{TR} / \mathrm{TE}[\mathrm{ms}]$ & $4000 / 101$ & $3.85 / 1.42$ & $4900 / 88$ \\
\hline Sequence type & Turbo-Spin-Echo & TWIST & EPI \\
\hline $\mathrm{FOV}[\mathrm{mm} \times \mathrm{mm}]$ & $200 \times 200$ & $260 \times 260$ & $204 \times 204$ \\
\hline Matrix & $320 \times 320$ & $160 \times 160$ & $136 \times 136$ \\
\hline Number of slices & $19 / 23 / 19$ & 20 & 20 \\
\hline Slice thickness [mm] & 3 & 3.6 & 3 \\
\hline Interslice gap [mm] & 0.3 & 0 & 0 \\
\hline Spatial resolution $\left[\mathrm{mm}^{3}\right]$ & $0.6 \times 0.6 \times 3.0$ & $1.6 \times 1.6 \times 3.6$ & $1.5 \times 1.5 \times 3.0$ \\
\hline b values $\mathrm{sec} / \mathrm{mm}^{2}$ & - & - & $0,50,500,800$ \\
\hline Flip angle $\left[{ }^{\circ}\right]$ & $137 / 150 / 150$ & 12 & - \\
\hline Parallel imaging Factor & Grappa (2) & Grappa (2) & Grappa (2) \\
\hline Temporal Resolution [sec] & - & 4.22 & - \\
\hline Averages & 4 & - & 8 \\
\hline Acquisition time [min] & $4.26 / 4.1 / 3.38$ & 5.02 & 5.4 \\
\hline
\end{tabular}

TWIST: Time-resolved angiography with interleaved stochastic trajectories; EPI: single-shot echo-planar imaging; TR: repetition time; TE: echo time; FOV: field of view.

courses observed in benign and malignant tissue can either be expressed semi-quantitatively by parameters derived from the gadolinium-concentration-time curves or quantitatively as Plasma Flow (PF) and Mean Transit Time (MTT) derived from pharmacokinetic models $(9,10)$.

DWI is used to measure the Brownian motion of water molecules in biological tissue. Due to the complexity of biologic tissues, actual diffusion coefficients of water protons cannot be measured directly. Thus, diffusion is measured indirectly by calculating a diffusion coefficient (the apparentdiffusion-coefficient, ADC) $(11,12)$. Although preliminary results have suggested a benefit to ADC and perfusion measurements for detection of ECE, no cut-off values have been established for quantitative discrimination between prostate carcinoma with and without ECE.

The purpose of this study was to evaluate the diagnostic performance of quantitative functional MR-parameters, such as ADC, PF and MTT for the assessment of ECE in PCa patients versus $\mathrm{T} 2 \mathrm{w}$ morphologic imaging.

\section{Patients and Methods}

Patients. A total of 34 patients (mean age 67 years, range $=52-78$ years) with pathologically proven PCa (Gleason score [GS] median 7 , range=6-9) were included in this retrospective analysis. The patients were referred for prostate MRI prior to RP by the department of urology between 12/2009 and 12/2012. Institutional review board (IRB) approval was obtained (2013-824R-MA) and written informed consent waived for this retrospective study. Every MRI exam was indicated clinically and no additional examination or therapy was performed.
A total of 4 patients in this series were part of a different study, previously published in Investigative Radiology (13), which focused on distinguishing PCa from prostatitis using DCE-MRI at $1.5 \mathrm{~T} v s .3$ T. Furthermore, all patients were part of a study published in European Journal of Radiology, comparing sonoelastography to MRI (14).

MRI protocol. MRI was performed on a 3T MR-system (Magnetom TimTrio, Siemens Healthcare Sector, Erlangen, Germany) utilizing a body as well as endorectal coil (Medrad Inc., Pittsburgh, PA, USA). The endorectal coil (ERC) was inserted after digital rectal examination and was inflated with $40-80 \mathrm{ml}$ of air. If not contraindicated, $40 \mathrm{mg}$ of $\mathrm{N}$-butylscopolaminium (Buscopan ${ }^{\circledR}$, Boehringer Ingelheim GmbH, Ingelheim, Germany) was injected intravenously to suppress bowel motion. The prostate MRI protocol consisted of a high-resolution T2-weighted triplane turbo-spin-echo (TSE) sequence, an axial, fat-suppressed, single-shot, echo-planar diffusion-weighted imaging (EPI-DWI) sequence, a 3D chemicalshift imaging MR spectroscopy (MRS), and an axial 2D T1weighted (T1w) dynamic contrast-enhanced (DCE) scan using spoiled gradient echo saturation recovery (Turbo FLASH).

The EPI-DWI sequence was acquired prior to gadolinium application and ADC maps were calculated automatically. The DCE-MRI examination was performed after bolus injection of 0.1 $\mathrm{mmol} / \mathrm{kg}(2.5 \mathrm{ml} / \mathrm{sec})$ body weight of gadolinium chelate (Dotarem ${ }^{\circledR}$, Guerbet, Roissy $\mathrm{CdG}$ Cedex, France). Imaging parameters of T2w, DCE, and DW sequences are detailed in Table I. Within 1 to 3 weeks after the MRI, all patients underwent RP. The prostate was histopathologically assessed by a pathologist with 7 years experience. The pathologist identified areas of PCa in a standardized manner with the prostate being divided into 16 parts and using the haematoxylin and eosin stain (H\&E stain). A histological capsular penetration was recognized if neoplastic glands were outside the prostatic capsular in the periprostatic tissue, according an ECE (14). 
Table II. Quantitative analysis of DCE MRI (PF, MTT) and DWI MRI (ADC) for ECE, CI and non ECE/CI cases.

\begin{tabular}{|c|c|c|c|c|c|c|}
\hline & \multicolumn{2}{|c|}{ ECE } & \multicolumn{2}{|c|}{ CI } & \multicolumn{2}{|c|}{ Non ECE or CI } \\
\hline & $\mathrm{PCa}$ & Normal tissue & $\mathrm{PCa}$ & Normal tissue & $\mathrm{PCa}$ & Normal tissue \\
\hline Mean PF & $37.7 \pm 25.0$ & $8.9 \pm 8.0$ & $22.0 \pm 12.3$ & $9.6 \pm 6.0$ & $25.82 \pm 41.4$ & $15.66 \pm 27.01$ \\
\hline Mean MTT & $68.8 \pm 22.9$ & $180.5 \pm 65.7$ & $102.9 \pm 43.5$ & $174.7 \pm 59.1$ & $87.23 \pm 40.8$ & $142.8 \pm 70.3$ \\
\hline Mean ADC & $655.4 \pm 151.3$ & $1736.8 \pm 257.8$ & $794.9 \pm 181.5$ & $1892.6 \pm 260.2$ & $906.8 \pm 260.2$ & $1926.2 \pm 318.9$ \\
\hline
\end{tabular}

ECE: Extracapsular extension; CI: capsular infiltration, PF: plasma flow (ml/100 ml/min); MTT: mean transit time (sec); ADC: apparent diffusion coefficient $\left(\mathrm{mm}^{2} / \mathrm{sec}\right)$.

Post-processing and Data-analysis. Post-processing of the DCEMRI data was performed by a radiologist (M.S.), with 4 years experience in prostate MRI, using an open source software tool for quantitative MRI perfusion analysis (UMM Perfusion, OsiriX DICOM viewer, Geneva, Switzerland) (15). The contrast enhancement curves were analyzed via a model-free deconvolution analysis as described previously $(16,17)$. The arterial input function (AIF) was measured in a plane wherein the common femoral artery was clearly opacified with the arrival of contrast agent and quantitative color-coded maps of PF and MTT were calculated $(16,17)$.

On the quantitative color-coded PF and MTT as well as ADC maps, a single region of interest (ROI) was drawn in areas with histologically proven $\mathrm{PCa}$ in concordance with the histopathological analysis scheme (see Figure 1). A second ROI was placed in a contralateral area without pathological findings.

For all ROIs PF, MTT and ADC values were measured as the mean of pixel values. A ROI volume of $0.3 \times 0.3 \mathrm{~cm}^{2}$ was used for all examinations. For the normalization and standardized comparison of PF, MTT, and ADC values among the different exams, ratios of these parameters in $\mathrm{PCa}$ and normal tissue were calculated in every patient.

Two other radiologists with 10 (D.J.D.) and 15 (S.O.S) years experience in prostate MRI separately evaluated the high-resolution triplane T2w-images for ECE. Both radiologists were aware of the diagnosis of PCa but were otherwise blinded to any clinical and pathological information. By definition, ECE was diagnosed on the triplane $\mathrm{T} 2 \mathrm{w}$-images if at least one of the following criteria were available: irregular capsular bulge, disruption of the prostatic capsule, extension into the periprostatic fat, obliteration of the recto prostatic angle, or asymmetry/involvement of the neurovascular bundle $(18,19)$.

Statistical analysis. Statistical analysis was performed with a statistical software package (SAS System, release 9.2, SAS Institute Inc., Cary, NC, USA). DWI- and DCE-MRI parameters are presented by their mean values and standard deviations; for ordinal scaled parameters median values are given. Kappa coefficients have been calculated to assess the strength of agreement between two readers. In order to compare the mean values of two groups, 2 sample $t$-tests have been used. For the comparison of GS, Cochran Armitage trend test has been applied. Spearman correlation coefficients have been calculated to find a relationship between GS and quantitative parameters. For diagnostic evaluations, logistic regression analyses have been performed. Areas under the curve (AUCs) have been compared using a test according to De Long et al. (20).

\section{Results}

All examinations were successfully completed without any adverse events. Pathologically, ECE was present in 14 patients, whereas capsular infiltration (CI) was found in 11 patients. The median GS was 7 in all patients.

On the triplanar T2w-sequences, ECE was correctly diagnosed in only 4 out of 14 patients by each of the two experienced readers resulting in sensitivity (SS) of $29 \%$ and specificity (SP) of $85 \%$ for both readers. Hence, there were 10 false negative cases and 3 false positive cases for ECE based upon the evaluation of $\mathrm{T} 2 \mathrm{w}$-sequences alone. The interobserver agreement was poor with $\mathrm{K}=0.1$. CI was detected by reader 1 (D.J.D.) with a SS/SP of $96 / 44 \%$ and by reader 2 (S.O.S.) with a SS/SP $68 / 44 \%$.

A total of 34 areas with $\mathrm{PCa}$ and 34 control areas were included in the ROI analysis. The mean values of PF, MTT and $\mathrm{ADC}$ in $\mathrm{PCa}$ and contralateral normal prostate tissue for all three groups (ECE, CI, no ECE/CI) are summarized in Table II.

Statistically significantly higher PF-ratios $(p<0.0001)$, lower MTT-ratios $(p<0.0001)$ and lower ADC-ratios $(p=0.02)$ were found in areas of PCa with ECE versus areas of PCa without. Furthermore, we found a positive correlation between PF-ratios and GS ( $\mathrm{r}=0.4517, p=0.0073)$. However, differences in MTT- and ADC-ratios with GS were not observed.

Receiver operating characteristic (ROC) analysis identified maximum areas-under-the-curve (AUC) for cutoff-ratios of 3.2 with PF, 0.51 with MTT and 0.46 with ADC (Figure 2). Using these ratios ECE could be diagnosed with SS/SP of $93 / 95 \%$ (PF), 100/80\% (MTT) and 86/50\% (ADC), respectively. The AUC of ECE cases was 0.98, 0.92, and 0.69 for PF, MTT and ADC-ratios, respectively.

Furthermore, there were statistically significant differences between tumors with and without $\mathrm{CI}$ regarding PF-ratios $(p<0.0001)$ and MTT-ratios $(p=0.0134)$, and in contrast, no significant difference regarding ADC-ratios could be identified $(p=0.1203)$. Logistic regression analysis revealed AUCs of 0.89 (PF-ratio), 0.80 (MTT-ratio) and 0.69 (ADCratio). Optimal cut-off-ratios were 2.56 for PF-ratio (SS/SP, $84 / 89 \%$ ) and 0.42 for MTT-ratio (SS/SP, 52/100\%). 

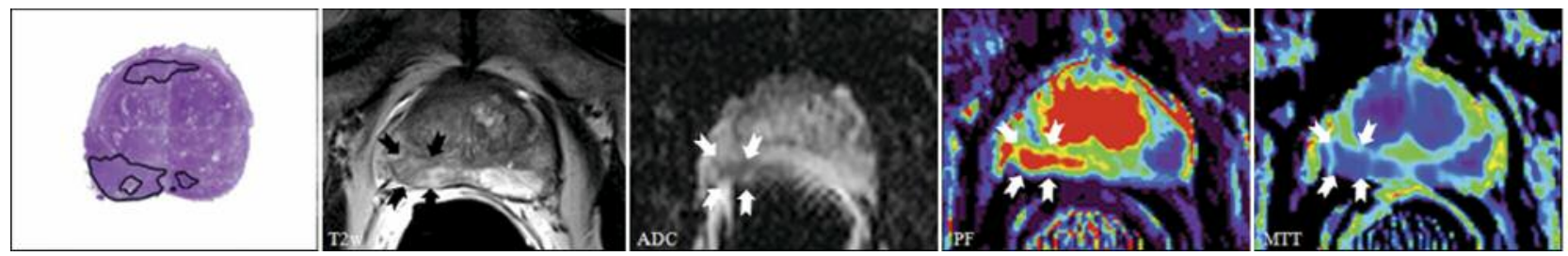

Figure 1. 69-year old man with PCa and ECE, Gleason score 7 (4+3), (a) axial histopathologic slice, pT3a (b) low signal intensity on the axial $T 2 w$ image without morphological signs of ECE and (c) restricted diffusion on the ADC map $\left(823.5 \mathrm{sec} / \mathrm{mm}^{2}\right.$, ratio* 0.40), DCE MRI demonstrates increased PF $(d)$ and decreased MTT (e) values/ratios (PF, $14.87 \mathrm{ml} / 100 \mathrm{ml} / \mathrm{min}$, ratio* 6.44; MTT 91.27 sec, ratio* 0.33$)$. *PCa/normal tissue.

a

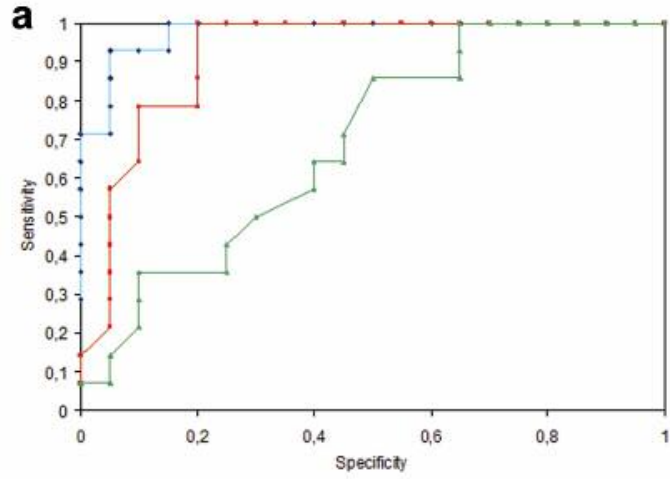

b

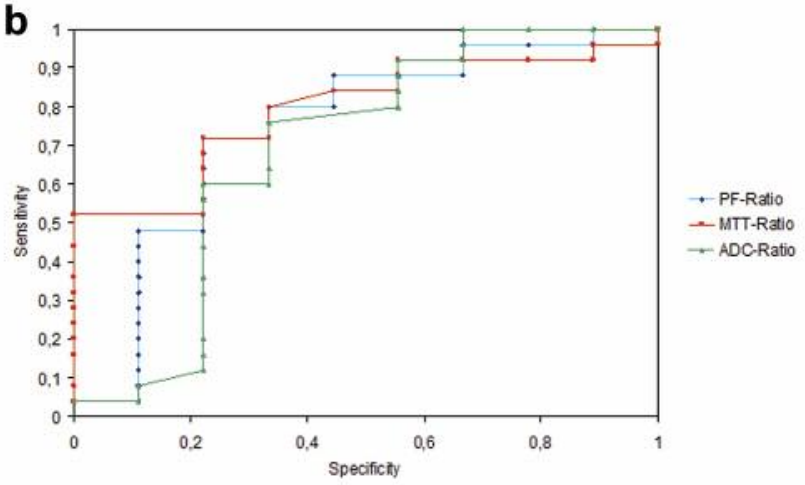

Figure 2. ROC (receiver operating characteristics) analysis. ROC analysis for detection a) of ECE (extracapsular extension) and b) of CI (capsular infiltration) with PF (plasma flow) ratios, MTT (mean transit time) ratios, and ADC (apparent diffusion coefficient) ratios. The AUC (area under the curve) of ECE/CI cases was 0.98/0.89, 0.92/0.80 and 0.69/0.60 for PF, MTT, and ADC ratios, respectively.

\section{Discussion}

This study demonstrates the successful identification of ECE and CI of PCa via use of quantitative DCE-MR parameters such as PF and MTT. In addition, ECE of PCa has been detected by quantitative ADC measurements. Both PF- and MTT-ratios successfully differentiated cases with and without ECE and CI. PF-ratios correlated with GS significantly; although, the relationship between MTT- or ADC-ratios and GS was not statistically significant. In distinction, the diagnostic performance of morphologic $\mathrm{T} 2 \mathrm{w}$-images was poor.

The reported SS and SP of T2w-sequences for the detection of ECE in PCa vary widely, ranging from $14 \%$ to $97 \%$, and this variation is a well-known limitation of the technique $(7,8)$. Reader experience is thought to play an important role in assessing ECE and SVI with MRI; however, Fütterer et al. demonstrated only incremental gains in SS/SP from 50 to $59 \% / 93$ to $96 \%$ for ECE in a study of 120 patients; SS for detection of SVI raised from 43 to $71 \%$ without change in SP (21). Similar to those findings, both readers in our study had more than 10 years experience in prostate MRI, however the SS and SP for ECE detection with high resolution, triplane $\mathrm{T} 2 \mathrm{w}$-images remained poor with no interobserver agreement. Moreover, cases of early ECE were frequently misclassified as CI. This mischaracterization was often related to known pitfalls in prostate MRI leading to misinterpretation (22), such as artifacts caused by the interposition of the faces of the ERC and the air filled rectum.

Numerous studies have demonstrated the added benefit of DWI-MRI for both localization as well as local staging of PCa $(23,24)$. These studies showed that ADC values, calculated from DWI measurements, correlate with both GS and local tumor aggressiveness. Moreover, low ADC values are a biomarker for the risk of recurrence following prostatectomy (24). In a recent study by Hara $\mathrm{T}$ et al. including $132 \mathrm{PCa}$ patients, ECE was diagnosed with respective accuracy and SP of $72 \%$ and $62 \%$ on ADC maps. However, even when all hypointense foci with capsular contact present on ADC maps were defined as representing ECE, the SS did not exceed 58\% (25). In our study, we also found statistically significant differences in ADC-ratios between cases with and without ECE, but not in cases with 
CI. Giganti et al. showed significant different ADC values and ADC-ratios in patients with and without ECE (26). The SS and SP for detection of ECE were moderate (SS/SP $85 / 50 \%$ ). The reason for this could be the magnetic susceptibility artifacts arising from the interface of the airfilled ERC and dorsal prostate tissue may have limited the robustness of ADC calculations for nearby lesions and decreasing diagnostic accuracy.

In the last decade several studies have demonstrated the benefit of DCE-MRI for diagnosis and localization of PCa, particularly in the detection of initial ECE in intermediate and high-risk patients (27). However, to the best of our knowledge, no study thus far has attempted a quantitative assessment of ECE with DCE-MRI. In a study by Bloch $e$ $a l$. with 32 PCa patients, a SS/SP of $91 / 86 \%$ was obtained utilizing a 1.5 T MRI-system and color-coded scheme of the DCE images. In that work, the authors defined the presence of extracapsular bright red pixel clusters larger than $3 \mathrm{~mm}$ in diameter as being positive for ECE (19). In a preliminary study by Fütterer et al. using a 3 T MRI-system, the criteria for ECE on DCE-MRI were: presence of asymmetric high peak enhancement, washout, and shorter onset time or an increased time-to-peak parameter. The fusion of parametric DCE and T2w-images resulted in a SS/SP of $65 / 95 \%$ versus $59 / 96 \%$ for the $\mathrm{T} 2 \mathrm{w}$-images alone (21). In a recent study by Min et al. the SS, SP, and accuracy of DCE-MRI for detecting ECE at 3 Tesla were, respectively, 27\%, 96\%, and $65 \%$. The relatively poor sensitivity reported in Min et al. may be related to the presence of post-biopsy hemorrhage. This was present in more than $90 \%$ of patients, since the time between biopsy and MR was 13.9 \pm 5.9 days. However, SS, SP, PPV, and NPV $(65 \%, 87.5 \%, 76.5 \%, 80 \%)$ did increase utilizing a combined evaluation including $\mathrm{T} 1 \mathrm{w}$, T2w, DCE, and DWI (28).

The key element of our study was the quantitative evaluation of the DCE-MRI parameters calculated by a deconvolution analysis $(9,15,16)$ which allowed differentiation between CI, ECE and non-ECE. As reported by Schlemmer et al. (29) an increased PF reflects a higher intratumoral microvessel density (MVD), which is considered a marker for neo-angiogenesis in malignant tissue. As further studies have described, MVD correlates with clinical/pathological stage, metastasis and histological grade, as well as disease-specific survival and progression after treatment $(30,31)$. In this context, we conclude that an increased MVD, as reflected by higher PF values and corresponding MTT shortening, also correlates with a higher probability of CI and ECE.

Our study has several limitations. For one, the relatively small number of patients limits the statistical relevance of the results. In addition, all examinations were performed using an air-filled ERC. Although this affords a greater signal-to-noise ratio, use of such a coil is associated with a number of disadvantages such as gland deformation and, especially for DWI, susceptibility-related artifacts. The latter may have contributed to the limited diagnostic accuracy observed with ADC calculations.

In conclusion, $\mathrm{CI}$ and ECE can be assessed by quantitative DCE-MRI analysis with a high-level of diagnostic confidence. Thus, quantitative DCE-MRI parameters have the potential to serve as a reader-independent risk estimator in the diagnosis of ECE, evaluating ECE with a greater SS and SP than ADC or T2-weighted image analysis. The statistical relationship between high GS and high PF also suggests that ECE correlates with increased MVD.

\section{Conflicts of Interest}

The Authors declare that there is no conflict of interest.

\section{References}

1 DeSantis CE, Lin CC, Mariotto AB, Siegel RL, Stein KD, Kramer JL, Alteri R, Robbins AS and Jemal A: Cancer treatment and survivorship statistics, 2014. CA Cancer J Clin 64(4): 252$271,2014$.

2 Heidenreich A, Bellmunt J, Bolla M, Joniau S, Mason M, Matveev V, Mottet N, Schmid HP, van der Kwast T, Wiegel T and Zattoni F: Aau guidelines on prostate cancer. Part i: Screening, diagnosis, and treatment of clinically localised disease. Actas Urol Esp 35(9): 501-514, 2011.

3 Thompson IM, Carroll PR and Carducci MA: Recommendations for defining and treating high risk localized prostate cancer. $\mathrm{J}$ Urol 176(6 Pt 2): S6-S10, 2006.

4 Tsurusaki T, Yamasaki Y and Maruta S: Outcome after radical prostatectomy with extended pelvic lymphadenectomy for untreated high-risk clinically localized prostate cancer. Nihon Hinyokika Gakkai Zasshi 104(3): 496-504, 2013.

5 Heidenreich A, Aus G, Bolla M, Joniau S, Matveev VB, Schmid $\mathrm{HP}$ and Zattoni F: Eau guidelines on prostate cancer. Eur Urol 53(1): 68-80, 2008.

6 Sohayda C, Kupelian PA, Levin HS and Klein EA: Extent of extracapsular extension in localized prostate cancer. Urology 55(3): 382-386, 2000.

7 Engelbrecht MR, Jager GJ, Laheij RJ, Verbeek AL, van Lier HJ and Barentsz JO: Local staging of prostate cancer using magnetic resonance imaging: A meta-analysis. Eur Radiol 12(9): 2294-2302, 2002.

8 Turkbey B, Albert PS, Kurdziel K and Choyke PL: Imaging localized prostate cancer: Current approaches and new developments. AJR Am J Roentgenol 192(6): 1471-1480, 2009.

9 Tofts PS, Brix G, Buckley DL, Evelhoch JL, Henderson E, Knopp MV, Larsson HB, Lee TY, Mayr NA, Parker GJ, Port RE, Taylor $\mathbf{J}$ and Weisskoff RM: Estimating kinetic parameters from dynamic contrast-enhanced $\mathrm{t}(1)$-weighted mri of a diffusable tracer: Standardized quantities and symbols. J Magn Reson Imaging 10(3): 223-232, 1999.

10 Buckley DL, Roberts C, Parker GJ, Logue JP and Hutchinson CE: Prostate cancer: Evaluation of vascular characteristics with dynamic contrast-enhanced t1-weighted $\mathrm{mr}$ imaging - initial experience. Radiology 233(3): 709-715, 2004. 
$11 \mathrm{Kim}$ CK, Park BK and Kim B: Diffusion-weighted mri at $3 \mathrm{t}$ for the evaluation of prostate cancer. AJR Am J Roentgenol 194(6): 1461-1469, 2010

12 Gupta RK, Sinha U, Cloughesy TF and Alger JR: Inverse correlation between choline magnetic resonance spectroscopy signal intensity and the apparent diffusion coefficient in human glioma. Magn Reson Med 41(1): 2-7, 1999.

13 Sertdemir M, Schoenberg SO, Sourbron S, Hausmann D, Heinzelbecker J, Michaely HJ, Dinter DJ and Weidner AM: Interscanner comparison of dynamic contrast-enhanced mri in prostate cancer: 1.5 versus 3 t mri. Invest Radiol 48(2): 92-97, 2013.

14 Pelzer AE, Heinzelbecker J, Weiss C, Fruhbauer D, Weidner AM, Kirchner M, Stroebel P, Schoenberg SO and Dinter DJ: Real-time sonoelastography compared to magnetic resonance imaging using four different modalities at $3.0 \mathrm{t}$ in the detection of prostate cancer: Strength and weaknesses. Eur J Radiol 82(5): 814-821, 2013.

15 Zollner FG, Weisser G, Reich M, Kaiser S, Schoenberg SO, Sourbron SP and Schad LR: Ummperfusion: An open source software tool towards quantitative mri perfusion analysis in clinical routine. J Digit Imaging 26(2): 344-352, 2013.

16 Sourbron S, Dujardin M, Makkat S and Luypaert R: Pixel-bypixel deconvolution of bolus-tracking data: Optimization and implementation. Phys Med Biol 52(2): 429-447, 2007.

17 Dujardin M, Sourbron S, Luypaert R, Verbeelen D and Stadnik T: Quantification of renal perfusion and function on a voxel-byvoxel basis: A feasibility study. Magn Reson Med 54(4): 841$849,2005$.

18 Tsuda K, Yu KK, Coakley FV, Srivastav SK, Scheidler JE and Hricak H: Detection of extracapsular extension of prostate cancer: Role of fat suppression endorectal mri. J Comput Assist Tomogr 23(1): 74-78, 1999.

19 Bloch BN, Furman-Haran E, Helbich TH, Lenkinski RE, Degani H, Kratzik C, Susani M, Haitel A, Jaromi S, Ngo L and Rofsky NM: Prostate cancer: Accurate determination of extracapsular extension with high-spatial-resolution dynamic contrastenhanced and $\mathrm{t} 2$-weighted $\mathrm{mr}$ imaging--initial results. Radiology 245(1): 176-185, 2007.

20 DeLong ER, DeLong DM and Clarke-Pearson DL: Comparing the areas under two or more correlated receiver operating characteristic curves: A nonparametric approach. Biometrics 44(3): 837-845, 1988.

21 Futterer JJ, Engelbrecht MR, Huisman HJ, Jager GJ, Hulsbergen-van De Kaa CA, Witjes JA and Barentsz JO: Staging prostate cancer with dynamic contrast-enhanced endorectal $\mathrm{mr}$ imaging prior to radical prostatectomy: Experienced versus less experienced readers. Radiology 237(2): 541-549, 2005.

22 Roethke MC, Lichy MP, Kniess M, Werner MK, Claussen CD, Stenzl A, Schlemmer HP and Schilling D: Accuracy of preoperative endorectal mri in predicting extracapsular extension and influence on neurovascular bundle sparing in radical prostatectomy. World J Urol 31(5): 1111-1116, 2013.
23 Hambrock T, Somford DM, Huisman HJ, van Oort IM, Witjes JA, Hulsbergen-van de Kaa CA, Scheenen T and Barentsz JO: Relationship between apparent diffusion coefficients at 3.0-t $\mathrm{mr}$ imaging and gleason grade in peripheral zone prostate cancer. Radiology 259(2): 453-461, 2011.

24 Park SY, Kim CK, Park BK, Lee HM and Lee KS: Prediction of biochemical recurrence following radical prostatectomy in men with prostate cancer by diffusion-weighted magnetic resonance imaging: Initial results. Eur Radiol 21(5): 1111-1118, 2011.

25 Hara T, Nakanishi H, Nakagawa T, Komiyama M, Kawahara T, Manabe T, Miyake M, Arai E, Kanai Y and Fujimoto H: Ability of preoperative 3.0-tesla magnetic resonance imaging to predict the absence of side-specific extracapsular extension of prostate cancer. Int J Urol 20(10): 993-999, 2013.

26 Giganti F, Coppola A, Ambrosi A, Ravelli S, Esposito A, Freschi M, Briganti A, Scattoni V, Salonia A, Gallina A, Deho F, Cardone G, Balconi G, Gaboardi F, Montorsi F, Maschio AD and De Cobelli F: Apparent diffusion coefficient in the evaluation of side-specific extracapsular extension in prostate cancer: Development and external validation of a nomogram of clinical use. Urol Oncol 34(7): 291.e9-291.e17, 2016.

27 Barentsz JO, Richenberg J, Clements R, Choyke P, Verma S, Villeirs G, Rouviere O, Logager V and Futterer JJ: Esur prostate mr guidelines 2012. Eur Radiol 22(4): 746-757, 2012.

28 Min BD, Kim WT, Cho BS, Kim YJ, Yun SJ, Lee SC and Kim WJ: Usefulness of a combined approach of t1-weighted, t2weighted, dynamic contrast-enhanced, and diffusion-weighted imaging in prostate cancer. Korean J Urol 53(12): 830-835, 2012.

29 Schlemmer HP, Merkle J, Grobholz R, Jaeger T, Michel MS, Werner A, Rabe J and van Kaick G: Can pre-operative contrastenhanced dynamic $\mathrm{mr}$ imaging for prostate cancer predict microvessel density in prostatectomy specimens? Eur Radiol 14(2): 309-317, 2004.

30 Weidner N, Carroll PR, Flax J, Blumenfeld W and Folkman J: Tumor angiogenesis correlates with metastasis in invasive prostate carcinoma. Am J Pathol 143(2): 401-409, 1993.

31 Brawer MK, Deering RE, Brown M, Preston SD and Bigler SA: Predictors of pathologic stage in prostatic carcinoma. The role of neovascularity. Cancer 73(3): 678-687, 1994. 